\title{
LA PRODUCCIÓN DE CONOCIMIENTO ETNOGRÁFICO A TRAVÉS DE LA INVESTIGACIÓN DE UN PROCESO MIGRATORIO: URUGUAYOS EN ARGENTINA
}

\section{The production of ethnographic knowledge through research of a migration process: Uruguayans in Argentina}

\section{ZULEIKA CROSA*}

\section{Fecha de recepción: 19 de enero de 2016 - Fecha de aprobación: 25 de septiembre de 2016}

\section{Resumen}

Este artículo propone destacar la importancia de explicitar la forma en que los antropólogos sociales producimos conocimiento acerca de la realidad social. Con este propósito realizamos un recorrido inicial sobre las bases epistemológicas y las consideraciones metodológicas que constituyen la investigación social para luego presentar y desarrollar los pasos del trabajo de campo que conforman la investigación antropológica. En esa dirección incluimos ejemplos de nuestra investigación sobre un proceso migratorio: el uruguayo en Argentina. En particular, el movimiento asociativo en sus distintas manifestaciones: políticas, sociales, ciudadanas y artísticas. De esta forma, la presentación de un modelo de investigación, propio de la Antropología Social, junto a las experiencias vividas a lo largo de nuestro estudio se complementan y conforman una trama inteligible que torna comprensible el quehacer de nuestra ciencia social.

Palabras clave: epistemología, metodología, trabajo de campo, procesos migratorios, uruguayos en Argentina

\section{Abstract}

This article aims to highlight the importance of explaining how social anthropologists produce knowledge about social reality. For this purpose, we make an initial route on the epistemological foundations and the methodological considerations that constitute social research and presents and develop the steps of the fieldwork that make anthropological research. In this direction, we include examples of our research on a migration process Uruguay in Argentina. The association movement in its various forms: political, social, civic and artistic. Thus, the presentation of a research model, typical of the Social Anthropology, with the experiences throughout our study complement and make an intelligible plot that makes understandable the work of our social science.

Keywords: epistemology, methodology, fieldwork, migration processes, uruguayans in Argentina

\footnotetext{
* Doctora y Licenciada en Ciencias Antropológicas. Universidad de Buenos Aires. Investigadora del Instituto de Ciencias Antropológicas (ICA) y docente del Departamento de Ciencias Antropológicas de la Facultad de Filosofía y Letras, Universidad de Buenos Aires, Buenos Aires, Argentina. Correo-e: zuleikacrosa@hotmail.com
} 


\section{Introducción'}

La pluralidad actual de perspectivas teóricas y conceptuales no impide la existencia de determinados consensos en las ciencias sociales. Por ejemplo, en Antropología Social, hay unanimidad en diversos aspectos. Cómo se produce conocimiento, cuáles son las características del mundo social y de la acción humana. También, los alcances y los límites de ciertas teorías como el positivismo, el empirismo, el racionalismo y la hermenéutica. Por último, es generalizado el conocimiento acerca la relación entre el lenguaje y la constitución del mundo social, por lo tanto, su importancia a la hora de producir conocimiento sobre fenómenos sociales.

Por su parte, es central en esa ciencia el trabajo de campo que incluye distintas situaciones específicas: el acceso, la observación participante, la descripción, el análisis y la escritura. Estos pasos conforman un entramado simultáneo en la práctica, aunque se pueden explicar por separado con fines expositivos.

Teniendo en cuenta la importancia de explicitar la forma en que los antropólogos sociales producimos conocimiento acerca de la realidad social realizamos este trabajo que incluye, en primer lugar, una presentación de las bases epistemológicas y las consideraciones metodológicas involucradas en la producción de conocimiento científico. Luego recorremos las particularidades del trabajo de campo, es decir, sus elementos constitutivos. Los presentamos como un proceso sincrónico, aunque sabemos que en la práctica se trata de un modelo diacrónico.
En todo momento intercalamos nuestra propia experiencia de construcción de un problema de investigación relacionado con las migraciones y con un proceso migratorio particular: el uruguayo en Argentina. Entendemos que la enumeración y explicación de los pasos que conforman la producción de conocimiento resultan comprensibles si los combinamos con las vivencias recopiladas por el investigador en cada situación, porque ambos constituyen una trama inteligible para comprender el quehacer antropológico. En síntesis, nos permiten mostrar como se construye un objeto de conocimiento y como toman cuerpo cada una de las instancias de la investigación social.

\section{Bases epistemológicas}

En la actualidad las ciencias sociales adscriben a orientaciones pluriparadigmáticas (Vázquez, 2000); cohabitan varias perspectivas conceptuales que articulan supuestos, categorías y criterios de validación del conocimiento para realizar interpretaciones sobre diversos aspectos de la realidad a considerar. Sin embargo, el mismo autor señala que las estrategias de investigación coinciden en priorizar la construcción de enunciados operativos, categorías, anticipaciones de sentido o hipótesis de trabajo adecuados a las problemáticas en cuestión y enumera ciertos acuerdos básicos que rigen la construcción de conocimiento desde una perspectiva histórica y crítica.

En la investigación social la teoría constituye un modelo operativo indisociable de la práctica. En ese entramado coexisten los juicios de realidad y de valor, y convergen de forma complementaria la interpretación y la explicación que resultan en concatenaciones de sentidos. Los 
sujetos, en este modelo, se conforman socialmente; pertenecen a un grupo o comunidad. Por último, el concepto de crítica resulta fundamental a la hora de implementar criterios de control epistemológico.

En otros términos, Rockwell (2009) coincide al remarcar la pluralidad conceptual contemporánea. Pero se ocupa de diferenciar su análisis, que caracteriza como histórico y etnográfico, de tres perspectivas cognitivas. El positivismo, que supuso una separación tajante entre el contexto de descubrimiento y el de verificación, entre el dato y la interpretación, entre la teoría y la descripción. Esta perspectiva pensó en un mundo social estático, constituido por fenómenos observables, y recurrió a procedimientos estandarizados de las ciencias naturales para lograr la objetividad.

El empirismo, marco de conocimiento que propuso un acceso directo, puro y ateórico a la realidad social o sus significados, mediante la escisión entre teoría y dato empírico y entre descripción, observación y análisis.

El racionalismo, que privilegió los métodos de conocimiento de carácter deductivo y formalista despreciando la investigación empírica y anulando el proceso mismo de construcción de conocimiento. Además, supuso la producción de conocimiento a partir de la total ruptura con las prenociones del investigador.

Con respecto a la tradición hermenéutica, si bien sustenta el trabajo de interpretación necesario para toda investigación social, su uso exclusivo o, en su defecto, su opocisión frente a otras tradiciones de conocimiento podría cerrar muchas vías de análisis, por ejemplo, el estudio de los procesos sociales y las relaciones de poder.
Por último, el carácter lingüístico del mundo social. El lenguaje cumple un papel importante en la producción de la realidad. Los términos que nombran determinados fenómenos, objetos, situaciones, etc., constituyen en su conjunto cosmovisiones de ese mundo social. Son clasificaciones que ordenan el pensamiento y dan sentido a las prácticas sociales. Existe un importante consenso sobre estos postulados en las ciencias sociales (Coulon, 1988; Butler, 2002).

Ahora bien, ¿en qué consiste la investigación social? En un sentido amplio, es la elucidación de lo naturalizado o constituido en el sentido común para recuperar el carácter histórico y procesual de las prácticas sociales que implican relaciones entre las personas. No se trata de eliminar el sentido común, sino de adjudicarle el valor de un material simbólico que puede servir de base a la producción científica (Nieto, 2007).

En particular, la investigación social promueve la descripción analítica de procesos particulares (que son parte de procesos generales) y requiere de la difícil labor, por parte del investigador, de construir criterios de análisis propios. Complementa el trabajo empírico y el conceptual, las construcciones de sentido de las personas o grupos, las negociaciones entre ambos mediante el diálogo y la reflexividad del investigador.

Entonces, ¿qué investiga la antropología? Prácticas sociales en la vida cotidiana (Heller, 1982) de personas y grupos de ellas en un contexto determinado, donde realizan interacciones en el marco de una sociedad heterogénea e interpretan su mundo de diferentes maneras de acuerdo con tradiciones históricamente construidas y valores institucionales. 
Estas orientaciones epistemológicas básicas conllevan derivaciones metodológicas que contemplaremos en el siguiente apartado.

\section{Consideraciones metodológicas}

Las prácticas sociales que intentamos conocer (a diferencia de los fenómenos naturales) se encuentran simbólicamente preconstruidas por partida doble (Giddens, 1982). Una interpretación proviene de los supuestos y categorías teóricas en contexto del investigador y otra, de los supuestos y clasificaciones de las personas que constituyen e interpretan su mundo social. Tanto uno como el otro, son intérpretes singulares y críticos; activos realizadores del mundo social (que actualizan las reglas del orden social y académico) y no meros portadores de estructuras (resisten y confrontan esos órdenes).

Comprender prácticas sociales preinterpretadas obliga al investigador a dialogar con las personas y a reflexionar acerca de su posicionamiento y de sus preconceptos en ese diálogo. Se trata de observar las prácticas cotidianas, entrar en diálogo con las personas y realizar un análisis que vincule esas prácticas con la historia que les dio forma.

En este proceso el antropólogo construye el dato etnográfico, definido por Batallan y García (1992) como un real construido, un material simbólico, un proceso de síntesis y atribución de sentido. Es la elaboración continua entre la interpretación que las personas brindan de su realidad en contexto y la reformulación del investigador con su marco teórico y conceptualizaciones. La acción del investigador adquiere cierta peculiaridadidad, ya que participa y/o pertenece a los mundos sociales que investiga, no es un extranjero sino que forma parte de la nación (siempre en formación) dentro de la cual trabaja y se encuentra posicionado en ella. Por ello, la política está en la reflexión de los antropólogos, aun si no la realizan ni la expresan como práctica. El ejercicio de la profesión es al mismo tiempo el ejercicio de la ciudadanía del investigador y de su compromiso, explícito o no, con la construcción de la nación (Cardoso de Oliveira, 1977).

Las personas no se encuentran pasivas frente a las condiciones estructurales que las predeterminan, tampoco son libres de construir estructuras ex nihilo ni son inherentemente teóricos sociales capaces de interpretar su conducta, intenciones, motivaciones, etc. Estos "procesos interpretativos requieren de un entrenamiento muy específico y complejo que no todas las personas realizan" (Vázquez, 2000: 29).

La reflexión y reformulación continuas del investigador implican explicitar las decisiones y los motivos que lo llevaron a una determinada construcción problemática de la realidad social. En este quehacer se encuentra entre dilemas epistemológicos y éticos propios de la época en la que vive e investiga, y debe tomar posición entre tradiciones de pensamiento en acuerdo con su perspectiva histórica, su visión del mundo, su concepto de ciencia y sus intereses conscientes e inconscientes de conocimiento (Vázquez, 2000).

La relación del investigador con las personas o grupos y el análisis descriptivo que resulta de la investigación antropológica, se constituyen mutuamente. Sin embargo, debemos considerar el modo en que las personas fueron producidas como tales, su identificación y clasificación; en términos de Foucault (1992), analizar el modo 
en que las relaciones de sujeción pueden fabricar sujetos. Aunque también es preciso incorporar la capacidad de agencia: las circunstancias cotidianas en las que los individuos confrontan con un rango de alternativas potenciales para la acción, lo cual implica que podrían haber actuado de otra manera (Giddens, 1982). Para que esto último suceda, los sujetos cuentan con una enorme variedad de modos prácticos de conocimiento (tácitos o inconcientes) sobre cómo proceder en los distintos contextos de la vida social.

Las bases epistemológicas y metodológicas reseñadas nos muestran que las prácticas sociales desarrolladas en la escala de la vida cotidiana son la parte visible de un conjunto de problemáticas que el antropólogo se propone construir sobre soportes empíricos y conceptuales. Esta construcción requiere en primer término indagar en el problema preconstruido para no ser el objeto de los problemas que se toman por objeto. Entendemos que la realidad social se constituye mediante instrumentos especialmente construidos; existe una "historia del trabajo social de construcción de instrumentos de construcción de la realidad social" (Bourdieu \& Wacquant, 1995: 172). Se pone en cuestión o problematizan las clasificaciones y los conceptos empleados para denominar los fenómenos a investigar. Conjuntamente, este trabajo implica la objetivación de los prejuicios, preconceptos y prenociones del bagaje académico y de sentido común académico.

Para realizar este ejercicio se requiere una formidable disposición crítica con respecto a los instrumentos de construcción de la realidad social, problemáticas, conceptos, técnicas y métodos.

\section{El comienzo de una investigación}

En nuestro trabajo de investigación (Crosa, 2014) comenzamos exponiendo el estado del conocimiento acerca de las migraciones y la forma en que ese objeto fue construido. Indagamos acerca de qué se habla y quiénes son los que hablan de las migraciones. Pudimos reconstruir procesos históricos, definiciones y formas de medición. También identificamos a los actores implicados; desde los propios migrantes y los Estados, hasta los organismos internacionales de cooperación y desarrollo.

En cuanto a nuestro interés de investigación, surgió particularmente de una cuestión autobiográfica que motivó preguntas acerca de las causas que inducen a grupos e individuos a migrar, los vínculos con el país de origen y de destino, y las modalidades de asociación y de vinculación entre compatriotas. El alto contenido biográfico, o el hecho de que el investigador sea parte del proceso migratorio que se propone conocer científicamente, implicaron una primera inmersión en los aspectos más conocidos intentando ordenar ese mundo habitual. Como antropólogos tenemos un saber previo que debemos objetivar y en cierta forma reordenar.

Comenzamos la investigación con el tema de la militancia política de los uruguayos en Argentina, que denominamos movimiento político. Las impresiones de la investigadora sobre esas prácticas se remontan a mediados de la década de 1980, en el barrio de Almagro (Ciudad Autónoma de Buenos Aires, Argentina), donde solía asistir en familia a las reuniones del comité de base denominado "19 de Marzo". Un comité de base en Argentina es un grupo de militantes del movimiento de coalición política Frente Amplio de Uruguay, que logró aglutinarse en torno a un nombre y a un espacio físico. 
Asimismo, fue testigo de la vida política de los uruguayos frenteamplistas en otros ámbitos. Nos referimos a las innumerables reuniones políticas donde se discutía, se estudiaba, se organizaban actividades proselitistas y se festejaba. En síntesis, los años de nuestra niñez y adolescencia, como uruguaya en Buenos Aires, transcurrieron siempre entre uruguayos comprometidos con la vida política de su país.

La primera formulación del tema o problema de interés quedó delimitada en torno a la centralidad del accionar político de los grupos migrantes en relación con su país de origen, y en particular de la colectividad uruguaya en Argentina.

Una vez lograda cierta conceptualización de lo conocido no solamente pudimos profundizar y complejizar el conocimiento previo, sino que avanzamos hacia otros aspectos del quehacer de algunos integrantes de este grupo. Esto es, facetas no observadas ni incluidas anteriormente como parte del problema de investigación: el movimiento social (asociaciones de residentes, casas culturales y grupos literarios), el movimiento ciudadano (propiciado por el Estado uruguayo mediante la formación de consejos consultivos de uruguayos en el extranjero) y el movimiento artístico (grupos carnavalescos).

Esta convivencia próxima implicó en nuestro caso repensar en profundidad las prenociones, que fueron constantemente interpeladas y puestas en cuestión a través de la observación participante y de los distintos contextos de entrevista. Solo así, despojándonos de nuestros ropajes, pudimos comprender el punto de vista de nuestros interlocutores (sus prácticas, sus creencias, sus intereses y sus deseos) más allá de los nuestros propios.
En esta primera parte de la investigación, pues, comenzamos a entender que los antropólogos somos nuestra propia herramienta de investigación y, por lo tanto, debemos calibrarnos para tal objetivo, proceso que atraviesa toda la investigación. En esta acción, comprendimos que somos un actor político más en ese mundo social, somos parte de él y por lo tanto el otro es muy cercano.

\section{El Trabajo de Campo}

Enumerar y desarrollar el conjunto de pasos elementales que conforman el trabajo de campo antropológico implica optar por una presentación operativa del tema. Sin embargo, dichos pasos se encuentran entramados y no siguen una secuencia lineal. Tampoco es unidireccional el derrotero de una investigación desde la epistemología y la metodología hasta el trabajo de campo. Pero la ficción del texto, que difiere de la práctica, nos permite transmitir al lector esta compleja temática en forma ordenada.

\section{La noción de campo}

El sentido de la noción de campo ha variado. En líneas generales, desde su forma tradicional entendida como un locus temporal y espacial acotado, hasta su redefinición como ámbito multisituado (Clifford, 1997). En particular, el concepto de campo remite a la construcción de un referente empírico y de un conjunto de relaciones sociales cuyo logro dependerá de la puesta en práctica de ciertos elementos del canon antropológico: la estancia prolongada, la comprensión de la lengua nativa, el acceso al punto de vista nativo, etc. (Malinowski, 1986). 
En nuestro caso hemos delimitado el análisis al movimiento asociativo de inmigrantes uruguayos en Argentina, teniendo en cuenta sus relaciones internas, con otros grupos de uruguayos en diferentes países, con la sociedad receptora y con el país de origen. Además indagamos en el conjunto de la producción impresa y virtual de estos grupos a los que tuvimos acceso. Nos referimos a comunicados, informes de opinión, revistas, gacetillas, folletos, propagandas, registros censales, correos electrónicos, etc. También incluimos fuentes de carácter estatal, como por ejemplo censos poblacionales y encuestas de hogares de Argentina y de Uruguay, debates parlamentarios en Uruguay, informes del Ministerio de Relaciones Exteriores de Uruguay, estatutos del Frente Amplio de Uruguay, informes del Instituto Nacional contra la Discriminación de Argentina y leyes migratorias de ambos países, así como fuentes supraestatales (los informes de las Conferencias Sudamericanas sobre Migraciones, la Organización Internacional para las Migraciones y las Naciones Unidas).

Analizamos el conjunto de relaciones dentro y entre los distintos grupos mencionados, con el conjunto de la colectividad uruguaya a quien van dirigidas sus acciones, participen o no del movimiento asociativo. Además, tomamos en cuenta, para la observación participante y el análisis, las relaciones establecidas con otros grupos en diferentes países del mundo y con distintos sectores sociales de Argentina, de Uruguay y con el Estado (instituciones diplomáticas y gubernamentales de ambos países). Con respecto a los organismos internacionales (supraestatales) si bien el vínculo con los grupos observados no fue directo, pudimos observar que los primeros influyen determinando lineamientos políticos sobre la cuestión migratoria que luego repercuten vía los Estados en los grupos de migrantes.

\section{El acceso}

Un punto esencial del trabajo de campo es el acceso a las personas que componen los ámbitos de estudio construidos por el investigador. Es crucial el tipo de relaciones que se logren establecer porque ellas determinarán el ingreso a ese mundo de sentidos (Nieto, 2007). El rol del antropólogo es central en este proceso; debe construir ciertas relaciones cara a cara, que no se diferencian demasiado de otros tipos de vínculos de la vida social. La diferencia con estos últimos es que las relaciones en el trabajo de campo deben ser cuidadas por el investigador, ya que de ello depende la continuidad de la investigación y el poder descubrir la perspectiva a través de la cual las personas dan sentido a su mundo social.

En los distintos ámbitos de nuestro trabajo de campo se presentaron situaciones variadas. Cuando comenzamos la investigación en el movimiento político lo hicimos a través de relaciones familiares. Es decir que la visión de ese mundo estuvo ligada a un sector político dentro del Frente Amplio de Uruguay constituido en Argentina.

La familiaridad nos abrió las puertas a un conjunto de actividades y relaciones que de otra forma no hubieran sido posibles. Sin embargo, nuestro rol de investigadores y el hecho de no ser militantes activos de ese sector político (asuntos aducidos y aclarados cada vez que fue necesario), nos cerraron el acceso a cierta información que solo incumbe a los miembros plenos de ese grupo. En efecto, en algunas 
ocasiones nos fue vedada la posibilidad de presenciar reuniones con políticos que venían de Uruguay. Pero en otras reuniones, también privadas, éramos especialmente invitados.

Esta forma de ingresar, además, nos quitó en su momento la posibilidad de relacionarnos con miembros de otros grupos partidarios o sectoriales, quienes nos habían posicionado políticamente por nuestro acceso (y no como investigadores). Así es como las personas tienden a incluir al investigador en su mundo y adjudicarle un rol conocido en él, dejándolo implicado de determinada manera (Althabe \& Hernández, 2005) y condicionándole la entrada a otros espacios (Berreman, 1962). Sin embargo, las alianzas políticas, en un frente de coalición partidaria y sectorial (como es el Frente Amplio de Uruguay) son muy dinámicas. El momento y el lugar del trabajo de campo marcarán los alcances y los límites del acceso.

Cuando trabajamos con el movimiento asociativo y ciudadano, fuimos interpelados por nuestra condición de nativos y puestos en la disyuntiva de tener que participar como miembros. Con límites precisos, lo hemos hecho. Por ejemplo, colaboramos en la confección de afiches, organización de eventos, presentaciones sobre el tema migratorio uruguayo y el voto en el exterior, en artículos escritos especialmente sobre la temática, en la venta y compra de bonos, rifas, libros, películas, etc. Pero no accedimos a ser miembros formales de ningún grupo. Evaluamos que dicha participación nos desplazaba de nuestro rol de investigadores y nos involucraba directamente en las problemáticas que imbuían a los miembros, ya fuera entre ellos o con la oficialidad del país de origen. ¿Cómo podíamos analizar una problemática social y entender los distintos puntos de vista si nos encontrábamos involucrados en las deliberaciones y decisiones? Nuestro límite nos cerró las puertas a cierta información relativa a los consejos consultivos. Se nos negó la clave para acceder a los foros virtuales semanales que realizaban los consejos consultivos de uruguayos conformados en varios países del mundo. Era una información importante que nos hubiera permitido tener una perspectiva más amplia y un conocimiento más acabado de los procesos que se estaban realizando al mismo tiempo en otros países.

De todas formas, nuestra decisión no impidió que emitiéramos opinión cada vez que fuimos interpelados, pero basados en ciertos fundamentos que nos daba nuestro trabajo de campo.

Sabemos que nuestra postura no es la única existente. A lo largo de nuestra investigación interactuamos con científicos sociales que aceptaron ser miembros del movimiento social y ciudadano uruguayo en otros países, aunque especificaron que esos tópicos no fueron su tema central de investigación.

Paradójicamente el ámbito más alejado de nuestra experiencia previa, basada en el conocimiento del movimiento político, fue el más amigable a la hora de desarrollar el trabajo de campo. Nos referimos al movimiento artístico en torno a las artes del carnaval, que incluían una murga (grupo artístico característico de la fiesta de carnaval), un corso o tablado (denominaciones utilizadas en Argentina y en Uruguay respectivamente para nombrar al espacio donde se festeja el carnaval), un taller de vestuario y maquillaje y un programa de radio.

Al comienzo lo sentimos un mundo extraño e incomprensible: reinaba el movimiento corpo- 
ral, el baile, la risa, el canto y sobre todo el juego grupal. Constantemente se jugaba a las adivinanzas, a inventar canciones, a hacer movimientos rítmicos simultáneos en grupo, etc. Era claro que estábamos entrando en el mundo de los artistas. Poco a poco fuimos moviéndonos y jugando con ellos mientras realizábamos nuestro trabajo de campo. También colaboramos en diversas tareas, como la cocina de alimentos y la confección del vestuario, y ofrecimos elementos (cuentas de colores, botones, etc.) siempre necesarios para el espectáculo artístico. Incluso, motu proprio, les regalamos discos compactos originales de murgas uruguayas. Pero en ningún momento fuimos requeridos para integrarnos a ninguna actividad, ayuda o colaboración.

Nuestro acceso a este proyecto artístico carnavalesco comenzó a través de sus miembros fundadores, quienes nos propusieron en primera instancia que asistiéramos a los ensayos semanales de la murga. Luego de concurrir en varias oportunidades, nos invitaron a las jornadas colectivas de confección del vestuario y de los ornamentos del espectáculo denominados fantasías. Entonces participamos de las comidas al mediodía. Después estuvimos en el programa de radio semanal. Allí por primera vez vivimos la experiencia de estar del otro lado del vidrio, de usar los audífonos, el micrófono y saludar al público oyente.

Otras experiencias por demás enriquecedoras con este grupo fueron los viajes a los distintos lugares donde realizaban sus espectáculos. Viajamos con los instrumentos y los trajes que ocupaban un espacio importante del ómnibus. Las salidas eran en horario de la tarde y durante el viaje se jugaba y se cantaba. Los regresos, después de medianoche, se daban entre conversaciones en voz baja y personas que dormían.

Durante dos años consecutivos asistimos al corso que realizan en el barrio de Almagro, en la Ciudad Autónoma de Buenos Aires. Llegábamos temprano para presenciar todos los preparativos y nos retirábamos a medianoche al finalizar el evento.

\section{Las presentaciones}

En directa relación con el acceso se desarrollan las presentaciones. El investigador se presenta a sí mismo y a su tema de investigación. A su vez, las personas presentan al investigador y al tema en distintos momentos y circunstancias. El carácter de las presentaciones es continuo y situado, su variación depende directamente del contexto (que incluye personas, ámbitos, etc.). Su importancia radica en las particularidades de la información obtenida y en el mantenimiento de los vínculos a futuro (fundamentales en el trabajo de campo prolongado).

A modo de ejemplo, en nuestro caso, asistimos a una reunión de directores de murga pertenecientes al circuito oficial del carnaval porteño (gentilicio que alude a los habitantes nacidos en la Ciudad Autónoma de Buenos Aires) acompañando a la directora de la murga, donde nos presentó, en todo momento, como la antropóloga. Fue el único contexto en el que fuimos antropólogos, en otros momentos fuimos periodistas, sociólogos, biólogos, nativos, familiares, hasta inspectores de la Sociedad Argentina de Autores y Compositores (SADAIC). En ocasiones nuestra presencia no fue acompañada de presentaciones explícitas. 
La presentación como antropóloga pudo implicar un subcampo o acuerdo preliminar no siempre explícito (Abélès, 2002), donde hubo un arreglo concreto en el que ambas encontramos un beneficio al situarnos en una posición jerárquica. Nunca fue dicho, pero constituyó una presentación determinante que legitimó la presencia de la investigadora en esa reunión y el prestigio de la directora. Cabe destacar que el grupo en cuestión valora en forma positiva la presencia de investigadores y estudiantes relacionados con las ciencias sociales. Más aun, incluye en su programa de radio una columna sobre temáticas relacionadas con la fiesta del carnaval a cargo de una antropóloga reconocida por su vasta trayectoria en la docencia y la investigación.

En síntesis, todas las formas de acceso y las modalidades de presentación fueron dinámicas y constantemente redefinidas por todas las personas implicadas. Incluso por el investigador que ante cada situación de trabajo de campo dice quién es y cuáles son sus intenciones; en distintos momentos, ante diferentes personas y hasta frente a las mismas personas en cada oportunidad.

\section{La observación y la descripción}

En vínculo estrecho con los pasos anteriores el investigador observa y describe. La observación participante sigue un plan orientado por el interés de investigación previamente esbozado que se nutre de las consideraciones epistemológicas y metodológicas ya mencionadas, pero también requiere cierta predisposición a registrar situaciones incomprensibles que probablemente puedan ser entendidas en los siguientes pasos del trabajo de campo, por ejemplo la entrevista.
La descripción de la realidad social requiere de una riqueza léxica (Lahire, 2006). Para imaginarnos esta situación el autor propone considerar una descripción en la cual el investigador, en un ambiente marítimo no utilice denominaciones específicas como borda, escotilla, popa, babor, estribor y nudos marineros. Este condicionamiento conduce a una descripción pobre. Asimismo, lo observado debe ser descrito atendiendo a la materialidad de los objetos, de los gestos y de las situaciones. Sin ser positivistas debemos detallar empíricamente los hechos observables y descriptibles, y producir informaciones confiables (verificables) sobre la realidad social. Estas informaciones no constituyen todo lo real "pero ese real seleccionado y construido puede ser empíricamente observado, verificado, y aquella interpretación que dejara de lado esa fase de constitución sería de muy poco interés" (Lahire, 2006: 40).

El acceso al mundo artístico, ya mencionado, luego de un encuentro casual que nos permitió tomar contacto con los miembros fundadores del grupo, comenzó con la observación de los ensayos de la murga en una plaza pública de la Ciudad Autónoma de Buenos Aires. Allí fuimos presentados como antropólogos realizando una investigación sobre los uruguayos en Argentina que culminaría con la elaboración de un libro, que efectivamente se concretó tres años después (Crosa, 2015). La precisión de esa presentación nos permitió realizar nuestro trabajo sin que las personas (aproximadamente treinta) tuvieran dudas sobre nuestro rol allí. Además, el ámbito era por demás apropiado. Estábamos en un lugar público y frente a personas habituadas a ser observadas. Nuestra presencia continua generó la confianza necesaria y pudimos ingresar a los restantes ámbitos de trabajo del grupo previa invitación. En una 
de esas ocasiones cuando participamos del taller de vestuario conversamos animadamente con uno de los miembros que se mostraba muy locuaz, mientras ayudábamos en la costura. De inmediato fuimos observados por las mujeres allí presentes quienes se ubicaron a nuestro alrededor interrumpiendo el diálogo. Fue una situación por demás extraña, que quedó registrada en nuestras notas de campo y pudimos develar meses después durante una entrevista.

\section{Las entrevistas}

El acceso, las presentaciones, la observación y las conversaciones ocasionales con las personas nos permitieron conocer las actividades, rutinas, jerarquías, modos de organización, jergas, etc. Pero este conocimiento resulta incompleto si no entramos en diálogo con las personas. Es necesario que nos expliquen qué hacen en sus propios términos. Cuáles son sus motivaciones, intereses, roles, expectativas e ideales, es decir, los puntos de vista. Aquí radica la diferencia entre una entrevista de carácter etnográfica y una encuesta; el investigador ya se encuentra inserto en el grupo y sabe, en cierta forma, qué y cómo preguntar. La entrevista presupone un modelo de interacción social que implica tener en cuenta las normas del entorno social y los modos apropiados de la interacción verbal específicos de cada ámbito, que en general son variables (Briggs, 1986; Guber, 2011). Dadas estas condiciones, los antropólogos sociales no comenzamos haciendo entrevistas como punto de partida de nuestra investigación. De ahí el trabajo que efectuamos previamente.

Por su parte, en el diálogo intervienen diversos factores. El lenguaje no sólo es referencial, debemos tener en cuenta el contexto en el cual lo dicho cobra sentido. De acuerdo con Briggs "el contexto en el que se emite una pregunta, a menudo, afecta la interpretación de la pregunta y la naturaleza de su respuesta" (1986: 4). Entonces nuestra idea es no solamente estar atentos al contexto sino además propiciar diversos contextos de entrevista para obtener información variada.

Siguiendo el planteo de Briggs (1986), los entrevistadores deben adquirir, más allá de la competencia lingüística (en su caso el autor debía hablar el idioma español), la competencia comunicativa. En otras palabras: además de la sintaxis y la semántica, saber qué expresiones pueden ser usadas en ciertas circunstancias para expresar ciertos significados o tener el dominio del uso de las propiedades indexicales del lenguaje (contexto, gestos, proxemia, prosodia, situación social, interlocutores presentes, etc.). Comprender los códigos nativos implica para el investigador antropólogo poder interactuar en el medio que investiga. Esto incluye el conocimiento de las formas de vestirse, las fórmulas de cortesía, la ubicación espacial adoptada en cada momento, la identificación de los roles entre los miembros, etc.

En una de las primeras entrevistas realizadas a la directora de la murga, la conversación versó casi exclusivamente en torno a las relaciones amorosas furtivas que surgen dentro del grupo y las personas detectadas por ella que propician ese tipo de relaciones. Según su punto de vista eran perjudiciales para la convivencia cotidiana y debían ser evitadas. En ese momento comprendimos la situación de alerta que había generado nuestro diálogo con un miembro del grupo (proclive, según supimos, a generar vínculos amorosos pasajeros) y la consecuente y explícita interrupción del mismo. 
Durante el transcurso del trabajo de campo distintas situaciones nos mostraron la dificultad de sostener el rol profesional cuando en todo momento se mezclaba, en los contextos de observación participante y entrevista, nuestra condición de mujer, sola, migrante, nativa, marcada política y familiarmente, etc.

Ahora bien, la entrevista en el estudio de procesos migratorios tiene sus particularidades. Hay un conjunto de preguntas insoslayables. Por ejemplo: motivos de la migración, año, edad, situación familiar, trabajo o profesión, nivel de instrucción, organizaciones o instituciones de pertenencia, perspectivas de regreso al país de origen, etc. Con ellas establecemos un perfil básico de las personas y su proceso migratorio.

Sin embargo, aunque comenzamos utilizando un guión pautado de entrevista que abarcaba algunos ejes principales, poco a poco fuimos comprendiendo que debíamos ser más flexibles y dejar que nuestros interlocutores contaran su historia en sus propios términos y secuencia. De esa forma podíamos obtener sus categorías sociales e interpretaciones y luego ponerlas en diálogo con nuestras propias categorías e interpretaciones como investigadores. Sólo así pudimos construir nuestras anticipaciones de sentido modificables y modificadas progresivamente a medida que avanzaba el proceso analítico. Nuestra preocupación inicial por obtener información básica no fue soslayada al adoptar una forma más flexible en las entrevistas, debido a que todos los temas que nos interesaban igualmente iban apareciendo en el relato.

Retomando la importancia del contexto en la entrevista, una respuesta recurrente a la pregunta por los motivos de la migración fue la militancia política desarrollada en Uruguay. En general, los entrevistados, priorizaron la militancia política entre las causas de la emigración. Mientras avanzaba la entrevista y al repreguntar, los interlocutores comenzaban a referirse a nuevos aspectos (falta de trabajo, alto costo de vida, la cercanía territorial y social con Buenos Aires) como otros desencadenantes de la migración. Esto da cuenta del valor dado a la actividad política en contexto de entrevista y el carácter secundario adjudicado a otras explicaciones de índole económica y geográfica. Desde nuestro punto de vista, la migración se encuentra motivada por un entramado muy complejo de causales que la determinan.

\section{El análisis y la escritura}

A nuestro criterio el análisis constituye uno de los puntos centrales de la investigación. Destacamos la imposibilidad de separarlo de la recolección de información, pues ambos se desarrollan en simultáneo. Nos distanciamos de las modalidades de investigación que parten de diseños precodificados y relegan el análisis a una instancia final. Por el contrario, según nuestra experiencia, el trabajo en terreno, la realización de los respectivos registros de campo, la consulta bibliográfica y el proceso de análisis (que involucra conceptualizaciones) se articulan y retroalimentan en forma constante.

En nuestro caso podemos trazar en líneas generales los ejes analíticos que fuimos articulando en torno a nuestro trabajo sobre el movimiento asociativo uruguayo en Argentina. 
El concepto que dio sentido a nuestro trabajo fue la idea de identidad y sus facetas interactivas, primordiales, constructivas e instrumentales (Bartolomé, 2006). Entendemos que la identidad es un proceso, se encuentra en movimiento, por ende es un devenir. Asimismo, lejos del marco positivista, comprendimos que podíamos acceder a las distintas manifestaciones de la identidad en tiempo y espacio. Analizamos como operó un descentramiento de la identidad política y cierta primacía de las manifestaciones identitarias sociales (basadas en elementos recreativos, patrióticos, literarios, etc.) en el marco de un importante cambio político en Uruguay; cuando se vislumbra la posibilidad del triunfo electoral del Frente Amplio a nivel nacional en ese país.

El análisis del movimiento ciudadano desplegado en Buenos Aires, en otras ciudades y países, a instancias de las políticas de vinculación promovidas desde el gobierno uruguayo a partir del año 2005, nos mostró el accionar transnacional estatal. Más allá de la relación siempre sostenida de los migrantes con su lugar de origen fuimos testigos de las respuestas estatales que esta relación generó y su impacto en los grupos migrantes, en especial en Buenos Aires.

La idea de integración fue central en el análisis del movimiento artístico. El circuito oficial del carnaval porteño fue un contexto propicio para mantener las tradiciones del carnaval montevideano, para incorporar elementos del carnaval porteño y a su vez para introducir de forma constante elementos nuevos. En la integración determinados elementos perduran otros se incorporan y también surgen novedades, hay un movimiento y una renovación constantes.
Por último, en nuestra experiencia de investigación destacamos el proceso de escritura como el más arduo; el que más tiempo nos ha llevado. Hay un momento en que debemos escribir buscando un "equilibrio en el relato de la experiencia, sin la pretensión de hablar por otros, pero con la convicción de tener algo que decir sobre lo que se aprendió entre ellos" (Rockwell, 2009: 202). La escritura reúne lo observado y el análisis de su devenir. De todas formas, solo una porción de la experiencia surge en la escritura.

Dicho de otra manera, la escritura etnográfica, entrelaza los procesos de escritura anteriores (notas de campo, registros de observación, trascripción de entrevistas, etc.). Se actualiza e integra lo investigado poniendo a prueba observaciones, categorías y razonamientos. En síntesis, escribir es continuar la investigación y es la fuente de nuevas preguntas (Cefai, 2013).

\section{Conclusiones}

Resulta esperable que los investigadores presenten los resultados de su trabajo una vez concluidos incluyendo un apartado, en general denominado metodológico, donde enumeren una serie de procedimientos básicos como la observación participante, la entrevista, la consulta y el análisis de fuentes de información. Sin embargo, no es habitual la realización de trabajos que expliciten la forma en que se construye el conocimiento sobre un fenómeno social. Por ejemplo, las decisiones tomadas, las interacciones con las personas, las situaciones incomprendidas, etc. Menos aun que se expliciten los lineamientos epistemológicos y metodológicos que guiaron la investigación. Los mismos suelen permanecer implícitos y queda por cuenta del lector su dilucidación. 
Teniendo en cuenta esta limitación, en este trabajo, propusimos presentar en forma ordenada los elementos que conforman el quehacer antropológico en su práctica cotidiana. Se trata de una reflexión que combina un modelo de investigación y el propio trabajo de investigación. En forma deliberada, optamos por no incluir elementos textuales (notas de campo, fragmentos de registros y entrevistas, etc.) que podrían resultar erráticos e incomprensibles para el lector. Por el contrario, decidimos presentar nuestro trabajo de campo mediado por nuestra propia reflexión y análisis sobre el mismo.

Explicitar las bases epistemológicas nos permitió situarnos frente al problema de la producción de conocimiento científico y encontrar los consensos actuales. La investigación social incluye la construcción de elementos que anticipan las problemáticas a estudiar. Trabajamos con enunciados operativos, categorías, anticipaciones de sentido o hipótesis. Por su parte, la tradición hermenéutica continúa vigente si se tiene en cuenta que las interpretaciones van acompañadas de un exhaustivo análisis sobre los procesos sociales y las relaciones de poder. Por último, el lenguaje es un factor constitutivo de la realidad social, forma parte de la construcción del mundo social.

Investigar es recuperar el carácter histórico y procesual de las prácticas sociales que implican relaciones entre los hombres y describir en forma analítica, construyendo criterios propios, los procesos particulares que son parte de los procesos sociales más generales. La Antropología Social se ocupa de las prácticas cotidianas en contexto que conforman la heterogeneidad social construida histórica e institucionalmente.
Mediante las consideraciones metodológicas pudimos comprender que existe una construcción de la realidad social previa a la investigación. En ella las personas son agentes activos, es decir, reproducen y producen el mundo social. El antropólogo construye el dato etnográfico; realiza una elaboración continua que incluye la interpretación que las personas brindan de su realidad en contexto y la reformulación a partir de determinados marcos teóricos y conceptualizaciones que forman parte de su bagaje académico. En esta tarea, el investigador, forma parte del mundo social que investiga y, por lo tanto, se encuentra comprometido políticamente en él.

El trabajo de campo constituye un entramado de prácticas simultáneas: la construcción de un campo empírico y de relaciones sociales, el acceso y las presentaciones que en ellos tienen lugar, la observación participante, la descripción analítica y la escritura. En su conjunto, estos pasos, van acompañados de una constante reflexión y reformulación por parte del investigador porque la realidad social sobre la cual se construye conocimiento es muy dinámica.

Ya mencionamos la importancia de incluir en el desarrollo de este trabajo las particularidades de nuestra propia investigación y de construir una trama inteligible que torne comprensible el quehacer antropológico. El lector pudo conocer nuestra problemática de investigación, las unidades de observación y análisis. También comprender cómo realizamos el recorte de nuestro objeto de estudio.

Esperamos que esta contribución refuerce la relevancia que pueden tener los trabajos abocados a explicitar un modelo de investigación en relación con un estudio concreto, para evaluar los resultados obtenidos.- 


\section{Notas}

${ }^{1}$ La investigación de la cual se desprende el artículo corresponde a la tesis doctoral desarrollada entre los años 2009 y 2014 , gracias al

\section{Referencias bibliográficas}

Abélès, M. (2002). "El campo y el sub campo". En Ghasarian, C. et al. De la etnografía a la antropología reflexiva. Buenos Aires: Ediciones del Sol.

Althabe, G. \& Hernández, V. (2005). "Implicación y reflexividad en Antropología”. En Hernández, V. et al. Etnografías globalizadas. Buenos Aires: Sociedad Argentina de Antropología.

Bartolomé, M. A. (2006). Procesos interculturales. Antropología política del pluralismo cultural en América Latina. Buenos Aires: Siglo XXI Editores.

Batallan, G. \& García, J. F. (1992). "Antropología y participación. Contribución al debate metodológico”. En Publicar en Antropología y Ciencias Sociales, № 1.

Berreman, G. (1962). "Behind Many Masks: Impresssion Management In a Himalayan Village". In Hindus of the Himalayas. Berkeley: University of California Press.

Bourdieu, P. \& Wacquant, L. (1995). Respuestas. Por uma antropologia reflexiva. México: Grijalbo.

Briggs, C. (1986). Learning How to Ask. A sociolinguistic appraisal of the role of the interview in social science research. Cambridge: University Press.

Butler, J. (2002). Cuerpos que importan. Sobre los limites materiales y discursivos del "sexo". Buenos Aires: Paidós.

Cefai, D. (2013). “¿Qué es la etnografía? Debates contemporáneos. Primera parte. Arraigamientos, operaciones y experiencias del trabajo de campo. Persona y Sociedad. Vol. XXVII, № 1.

Cardoso de Oliveira, (1977). "Articulación inter-étnica en Brasil". En Hermitte, E. \& Bartolomé, L. (Comps.) Procesos de articulación social. Buenos Aires: Amorrortu. apoyo de la beca ofrecida por el programa de Doctorado en Ciencias Antropológicas de la Universidad de Buenos Aires.

Coulon, A. (1988). La etnometodología. Madrid: Cátedra.

Clifford, J. (1997). Itinerarios transculturales. Barcelona: Gedisa.

Crosa, Z. (2014). "Uruguayos en Argentina: el devenir de una identidad. Configuraciones identitarias, procesos nacionalistas y migraciones internacionales". Tesis de Doctorado en Ciencias Antropológicas. Buenos Aires: Universidad de Buenos Aires. (2015). Uruguayos en la Argentina. El devenir de una identidad. Buenos Aires: Biblos.

Giddens, A. (1982). "Hermeneutics and social theory". In Profiles an critiques in social theory. California: University of California Press.

Guber, R. (2011). La etnografía. Método, campo y reflexividad. Buenos Aires: Siglo XXI Editores.

Foucault, M. (1992). Genealogía del racismo. De la Guerra de razas al racismo de Estado. Madrid: Ediciones la Piqueta.

Heller, A. (1982). Sociología de la vida cotidiana. Barcelona: Ediciones Península.

Lahire, B. (2006). "Describir la realidad social". En El espíritu sociológico. Buenos Aires: Manantial.

Malinowski, B. (1986). Los Argonautas del Pacifico Occidental. Barcelona: Planeta Agostini.

Nieto, G. (2007). La inmigración china en España. Una comunidad ligada a su nación. Madrid: Catarata.

Rockwell, E. (2009). La experiencia etnográfica. Historia y cultura en los procesos educativos. Buenos Aires: Paidós.

Vázquez, H. (2000). Procesos identitarios y exclusión sociocultural. La cuestión indígena en Argentina. Buenos Aires: Biblos. 
